\title{
EDITORIAL
}

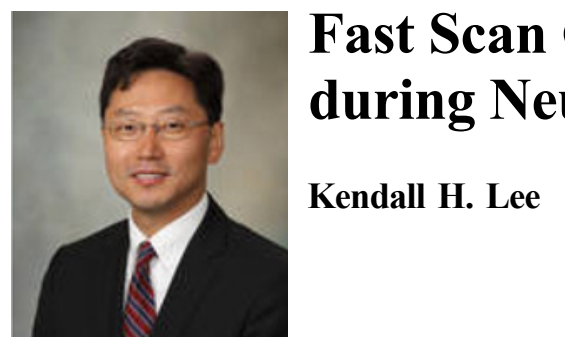

\section{Fast Scan Cyclic Voltammetry Recordings in Humans during Neurosurgery: Challenges and Opportunities}

(C) The Korean Society of Medical \& Biological Engineering and Springer 2011

It is with great pleasure to congratulate the launch of the premier journal of "Biomedical Engineering Letters", which is an official journal of the Korean Society of Medical and Biological Engineering. Biomedical engineering technology is one of the most crucial components in the interdisciplinary and translational research area. I believe this era will be the golden age of biomedical technology, applying the most advanced applications to scientific research. The launch of Biomedical Engineering Letters reflects this vision, and with its strong editorial board it will play a leading role opening up the biomedical engineering's last frontier.

Here, I briefly introduce the novel biomedical method of deep brain stimulation (DBS). DBS has been demonstrated to be an effective neurosurgical treatment for many neurological and psychiatric disorders including Parkinson's disease, tremor, epilepsy, depression, and chronic pain. We have developed a novel intraoperative neurochemical monitoring device called wireless instantaneous neurotransmitter concentration sensor (WINCS) system, which combines digital telemetry with amperometry and fast-scan cyclic voltammetry (FSCV) for real-time neuro-chemical measurements at an implanted microelectrode. WINCS could detect neurotransmitters such as dopamine, adenosine, serotonin, glutamate, and histamine with micro second temporal resolution and high sensitivity. We have now utilized the system in rats, pigs, and in human Parkinson's Disease (PD) and Essential Tremor (ET) patients during DBS neurosurgery.

WINCS hardware is a micro-processor controlled, MRIcompatible, battery-powered instrument that combines Bluetooth ${ }^{\circledR}$ digital telemetry with FSCV and constant potential amperometry. The WINCS base-station software

Kendall H. Lee $(\otimes)$

Neural Engineering Lab, Department of Neurologic Surgery, Mayo Clinic, Rochester, MN

Tel : +507-284-2511

E-mail : Lee.Kendall@mayo.edu
(WINCSware) controls the wireless patient module and processes the received data stream, and displays the results in nearly real time. The WINCS Electrode (WINCStrode) is based on an approved human extracellular tungsten electrophysiology electrode that was modified by the addition of a short section of carbon-fiber to enable FSCV recordings. To date, the WINCS hardware, WINCSware, and WINCStrode were used in rats, pigs, and in 15 human PD or ET Patients during clinical DBS neurosurgery. Leksell Model G Stereotactic headframe, MRI guided neuronavigation system was used for surgical targeting. Our protocol was approved by the Mayo Clinic IRB for human studies.

Successful real-time analysis of wireless FSCV recordings from WINCS using the WINCStrode were performed both in a flow cell in vitro, rats, pigs, and in human PD and ET patients undergoing DBS neurosurgery. For FSCV, neurochemical data were transmitted by the WINCS hardware to the computer base station for presentation of a continuous three-dimensional color plot of sequential backgroundsubtracted voltammograms. Analysis of the voltammograms revealed signals consistent with measurements of dopamine efflux when the WINCStrode was placed in the caudate and adenosine efflux when placed in the VIM thalamus. Following WINCS recordings, the patients had good clinical response from the bilateral STN or VIM DBS electrodes, without complication.

Our studies represent the successful feasibility and safety study using WINCS in animals and in humans. We believe the combination of these sophisticated in vivo techniques will provide important new insights into the neurobiological mechanisms of DBS action. Further, our results suggest that next generation DBS systems that couples digital telemetry with FSCV may be useful as the sensing component of a "smart" DBS device providing enhanced utility to human patients. 